\title{
Frequency Dependence and Cooperation: Theory and a Test with Bacteria
}

\author{
Adin Ross-Gillespie, ${ }^{1, *}$ Andy Gardner, ${ }^{1,2, \dagger}$ Stuart A. West, ${ }^{1, \neq}$ and Ashleigh S. Griffin ${ }^{1, \$}$
}

1. Institute of Evolutionary Biology, School of Biological Sciences, University of Edinburgh, King's Buildings, Edinburgh EH9 3JT, United Kingdom;

2. St. John's College, Oxford University, Oxford OX1 3JP, United Kingdom

Submitted December 4, 2006; Accepted April 23, 2007;

Electronically published July 19, 2007

AвSTRACT: Hamilton's inclusive fitness theory provides a leading explanation for the problem of cooperation. A general result from inclusive fitness theory is that, except under restrictive conditions, cooperation should not be subject to frequency-dependent selection. However, several recent studies in microbial systems have demonstrated that the relative fitness of cheaters, which do not cooperate, is greater when cheaters are rarer. Here we demonstrate theoretically that such frequency-dependent selection can occur in microbes when there is (1) sufficient population structuring or (2) an association between the level of cooperation and total population growth. We test prediction (2) and its underlying assumption, using the pathogenic bacterium Pseudomonas aeruginosa, by competing strains that produce iron-scavenging siderophore molecules (cooperators) with nonproducers (cheaters) at various ratios, under conditions that minimize population structuring. We found that both the relative fitness of cheaters and the productivity of the mixed culture were significantly negatively related to initial cheater frequency. Furthermore, when the period of population growth was experimentally shortened, the strength of frequency dependence was reduced. More generally, we argue that frequency-dependent selection on cooperative traits may be more common in microbes than in metazoans because strong selection, structuring, and cooperation-dependent growth will be more common in microbial populations.

Keywords: Pseudomonas aeruginosa, public goods, siderophores, strong selection, kin selection, population structure.

\footnotetext{
* E-mail: a.ross-gillespie@ed.ac.uk.

† E-mail: andy.gardner@ed.ac.uk.

¥ E-mail: stu.west@ed.ac.uk.

`E-mail: a.griffin@ed.ac.uk.
}

Am. Nat. 2007. Vol. 170, pp. 331-342. () 2007 by The University of Chicago. 0003-0147/2007/17003-42256\$15.00. All rights reserved.

DOI: $10.1086 / 519860$
Explaining cooperation is one of the greatest challenges for evolutionary biology (Maynard Smith and Szathmàry 1995; Hamilton 1996). The problem is this: why should an individual perform a cooperative behavior that appears costly to perform but benefits other individuals (Hamilton 1964)? Following a wealth of theoretical work in this area in recent decades, we now have an excellent general understanding of the different ways in which cooperation can be favored (reviewed by Frank [2003]; Sachs et al. [2004]; Lehmann and Keller [2006]; West et al. [2006], [2007]). However, much of this vast body of theory has been developed to elucidate general principles and is less useful for stimulating empirical tests of theory (Leimar and Hammerstein 2006). Consequently, a major task for social evolution research is to take this general theory and develop it so it can be tested empirically, with specific groups of organisms.

Microbes offer a number of advantages for testing social evolution theory (reviewed by Crespi [2001]; West et al. [2006]). In particular, they perform a number of cooperative behaviors, where it is possible to alter the costs and benefits of cooperation experimentally and then follow the fitness consequences (West et al. 2006). Recently, there has been interest in how the relative fitness of cooperators, and cheaters who do not cooperate (or cooperate less), depends on the relative proportion of cooperators in the population (Levin 1988; Velicer et al. 2000; Dugatkin et al. 2003, 2005; Harrison et al. 2006; MacLean and Gudelj 2006). However, these experimental examinations of frequency dependence contrast with most theory in this area, where frequency dependence is not predicted. Indeed, a major result from Hamilton's (1964) original inclusive fitness formulation, showing how cooperation can be favored between relatives, was that the inclusive fitness of cooperators did not vary with gene frequency at all. Instead, because increased cooperation provides benefits to both cooperators and cheaters, the inclusive fitness advantage of cooperation remains constant despite changes in the frequency of cooperators. Hamilton was so pleased by this 
result that he later described it as a "gift from God" (Hamilton 1988 , p. 16). Subsequent work has shown that frequency dependence can enter into kin selection theory in cases featuring strong selection (genes with large fitness effects) or nonadditivity (e.g., Charlesworth 1978; Michod 1982; Toro et al. 1982; Queller 1984; Day and Taylor 1998; Frank 1998; Rousset 2004; Wild and Traulsen 2007).

Our first aim in this article is to develop theory that predicts specifically when and why frequency-dependent selection on cooperation should be observed in microbes. The phenomenon is illustrated by considering the extracellular secretion of substances that can be utilized by neighboring cells. The production of such "public goods" requires explanation because they are costly to the individual to produce but provide a benefit to the local group (West et al. 2006). In this situation, we might, at first glance, expect that selection on cells that contribute less than their fair share to the public good resource pool (cheaters) should be frequency dependent. That is, cheaters should do better when they are rare, because there will be more public goods/cooperators for them to exploit. However, cooperators also gain a benefit from being in a more cooperative population. In the simplest scenario, these two effects exactly cancel, and so selection on cheaters and cooperators does not depend on their frequency (see next section). We examine the extent to which frequency dependence does arise when the following biological complexities are taken into consideration: population structure, effects of cooperation on population growth, and large (as opposed to small) fitness consequences associated with variation in the level of cooperation (strong selection).

Our models predict that, given strong selection, cheaters will be subject to negative frequency-dependent selection (i.e., their relative fitness will be lower when common) when (1) the population is structured and/or (2) a higher frequency of cooperators leads to greater population growth. We tested prediction (2) and the underlying assumption on the relationship between growth and cooperator frequency by investigating the production of a public good, iron-scavenging siderophore molecules, in the bacterial pathogen Pseudomonas aeruginosa (West and Buckling 2003; Griffin et al. 2004). Iron is a major limiting factor for bacterial growth, because most iron in the environment is in the insoluble Fe(III) form and is actively withheld by hosts (Guerinot 1994; Ratledge and Dover 2000). Siderophores scavenge insoluble and host-bound iron, making it available for bacterial metabolism. To determine whether frequency dependence is a general characteristic of $P$. aeruginosa siderophore production and not just a pleiotropic effect associated with a specific cooperator-cheater pair, we examined three independently derived cooperator-cheater strain pairs. These involved mu- tants produced by ultraviolet mutagenesis, single-gene deletion, and spontaneous natural mutation, respectively.

\section{Models and Analyses}

We explore some ways in which frequency-dependent selection can arise in kin selection models for the production of public goods dilemmas in microbes. In particular, we examine the role of (1) population structure (including mixing rates and the scale over which social interaction occurs) and (2) the dependence of population carrying capacity and growth on the level of public goods contributions. In both cases, we allow for strong selection, which is crucial for frequency dependence in the context of social behaviors and is an important characteristic of many microbial cooperative traits (see "Discussion"). To begin, we demonstrate that frequency dependence does not emerge under the simplest possible scenario with weak selection.

\section{Weak Selection and Frequency Independence}

In a broad class of models, weak selection leads to frequency independence (Rousset 2004, p. 80; 2006). To see why, consider a large population facing a public goods dilemma, such as, for example, siderophore production. An individual's fitness is a function of (1) its level of public goods contribution, $s$, and (2) the average public goods production across the whole population, $\bar{s}$. Without making further assumptions, we can express fitness as $w(s, \bar{s})$; this can be done for structured and unstructured populations. Further, we assume that cooperative individuals contribute an amount $s_{\mathrm{C}}$ and that a proportion $p$ of the individuals in the population are defectors (cheaters) who contribute a smaller amount, $s_{\mathrm{D}}=s_{\mathrm{C}}-\delta$; hence, $\bar{s}=$ $s_{\mathrm{C}}-p \delta$. Thus, the fitness of a cooperator is

$$
w_{\mathrm{C}}=w\left(s_{\mathrm{C}}, s_{\mathrm{C}}-p \delta\right)
$$

and the fitness of a cheater is

$$
w_{\mathrm{D}}=w\left(s_{\mathrm{C}}-\delta, s_{\mathrm{C}}-p \delta\right)
$$

The relative fitness of cheaters is given by the ratio of cheater and cooperator fitness and, using a Taylor expansion, can be expressed as

$$
v_{\mathrm{D}}=\frac{w_{\mathrm{D}}}{w_{\mathrm{C}}}=1-\left.\frac{\partial w}{\partial s}\right|_{\delta=0} \delta / \hat{w}+O\left(\delta^{2}\right)
$$

where $\hat{w}=w\left(s_{\mathrm{C}}, s_{\mathrm{C}}\right)$ is the fitness of cooperators in the absence of cheaters. This reveals that, to leading order in $\delta$, the relative fitness of cheaters is not a function of their 
frequency $p$ in the population (the partial derivative appearing in eq. [3] is not a function of $p$, because it is evaluated in the neutral population where $\delta=0$, so that cooperators and defectors are indistinguishable and the frequency of the latter is irrelevant). Hence, if selection is weak ( $\delta$ sufficiently small for higher-order terms in $\delta$ to be negligible; the $\delta$-weak selection of Wild and Traulsen [2007]), it is also frequency independent. For stronger selection, the trailing term of order $\delta^{2}$ becomes more important, and if this is a function of the frequency of cheaters, then there is the possibility of frequency-dependent selection. Note that although our analyses focus on whether the fitness of cheaters is frequency dependent, the fact that we are examining relative fitness means that they also demonstrate the nature of selection on cooperatorsif the relative fitness of cheaters decreases as they become more common, then the relative fitness of cooperators must increase as they become rarer.

\section{Structured Populations}

One way in which strong selection can give rise to frequency-dependent selection is if populations are structured so that cooperators and cheaters do not share the same social environment. This could occur in populations with localized social interaction due to limited mixing of public goods or cells. A consequence of this is that more cooperative individuals would have greater access to public goods, either because they enjoy preferential access to the public goods that they have produced or because they use the public goods produced by their clonal relatives (who are also cooperators). We examine this in general terms, describing fitness as a product of two components that capture (1) the cost of producing public goods and (2) the benefit of receiving public goods. In particular, we write

$$
w(s, \hat{s})=g(s) h(\hat{s}),
$$

where $w$ is fitness, $g$ is a growth component that decreases with one's individual allocation to public goods production $s$, and $h$ is a growth component that increases with one's access to public goods $\hat{s}$ (for related microbial models, see Brown 1999; Smith 2001; West et al. 2002; Dugatkin et al. 2003; West and Buckling 2003). In mathematical terms, we have $g>0, \quad \partial g / \partial s=g^{\prime}<0, \quad h>0, \quad$ and $\partial h / \partial \hat{s}=h^{\prime}>0$. We make the further assumption of linear or diminishing returns to fecundity as access to public goods in the social environment is increased, giving $\partial^{2} h / \partial \hat{s}^{2} \leq 0$. Due to population structure, the level of public goods available in the social environment is correlated with the individual's own allocation to public goods, according to the expression $\hat{s}=r s+(1-r) \bar{s}$; that is, the level of public goods available lies between one's own investment $(s)$ and the population average investment $(\bar{s})$, according to the relatedness between social partners $(r)$. The variable $r$ encapsulates the effects of both bacterial dispersal and the diffusion of public goods through the population. It therefore allows us to move between the extremes of a completely mixed population or global diffusion of public goods $(r=0)$ and a completely structured population, where all neighbors are of the same genotype or public goods disperse over such small distances that they are utilized only by the individual that produced them $(r=1$; cheaters only interact with cheaters, and cooperators only interact with cooperators).

We compare the fecundity of a cheater strain that allocates nothing to public goods ( $s=0$, defection) to that of a strain that allocates a standard unit $(s=1$, cooperation). Respectively, these are

$$
w_{\mathrm{D}}=g(0) h[(1-r)(1-p)]=g_{\mathrm{D}} h_{\mathrm{D}}
$$

and

$$
w_{\mathrm{C}}=g(1) h[r+(1-r)(1-p)]=g_{\mathrm{C}} h_{\mathrm{C}},
$$

where $p$ is the population frequency of cheaters, and hence $\bar{s}=1-p$ is the population average allocation to public goods, being the proportion of cooperators. The relative fitness of cheaters can be written as

$$
v=\frac{w_{\mathrm{D}}}{w_{\mathrm{C}}}=\frac{g(0) h[(1-r)(1-p)]}{g(h) I[r+(1-r)(1-p)]}=\frac{g_{\mathrm{D}} h_{\mathrm{D}}}{g_{\mathrm{C}} h_{\mathrm{C}}} .
$$

We now determine whether and how the relative fitness of cheaters $(v)$ varies with their frequency in the global population $(p)$ :

$$
\frac{\mathrm{d} v}{\mathrm{~d} p}=\frac{g_{\mathrm{D}}}{g_{\mathrm{C}}}(1-r) \frac{h_{\mathrm{D}} h_{\mathrm{C}}^{\prime}-h_{\mathrm{C}} h_{\mathrm{D}}^{\prime}}{h_{\mathrm{C}}^{2}} .
$$

This reveals the following: (1) in the absence of population structure $(r=0)$, there is no frequency dependence $(\mathrm{d} v / \mathrm{d} p=0)$, because cooperators and cheaters share the same social environment; hence, $h_{\mathrm{C}}=h_{\mathrm{D}}$ and $h_{\mathrm{C}}^{\prime}=h_{\mathrm{D}}^{\prime}$; (2) in fully structured populations $(r=1)$, there is no frequency dependence $(\mathrm{d} v / \mathrm{d} p=0)$, because an individual's social environment is dependent only on its own cooperation strategy and not on the global frequency of cooperators; (3) in a population with intermediate structuring $(0<r<1)$, there is negative frequency dependence $(\mathrm{d} v / \mathrm{d} p<0)$, because cooperators enjoy more public goods in their social environment than do cheaters and hence $h_{\mathrm{C}}>h_{\mathrm{D}}$ and $h_{\mathrm{C}}^{\prime} \leq h_{\mathrm{D}}^{\prime}$.

The impact of population structure $(r)$ on cheater rel- 
(A)

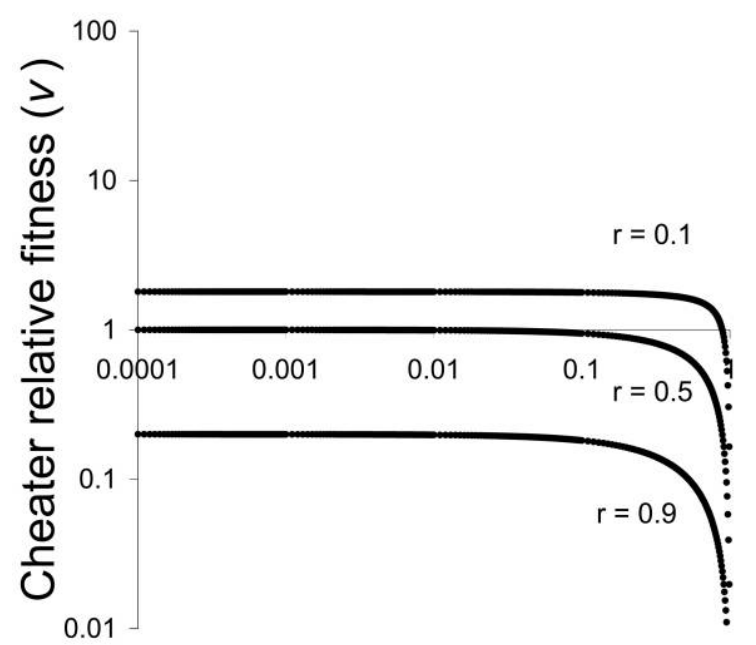

(B)

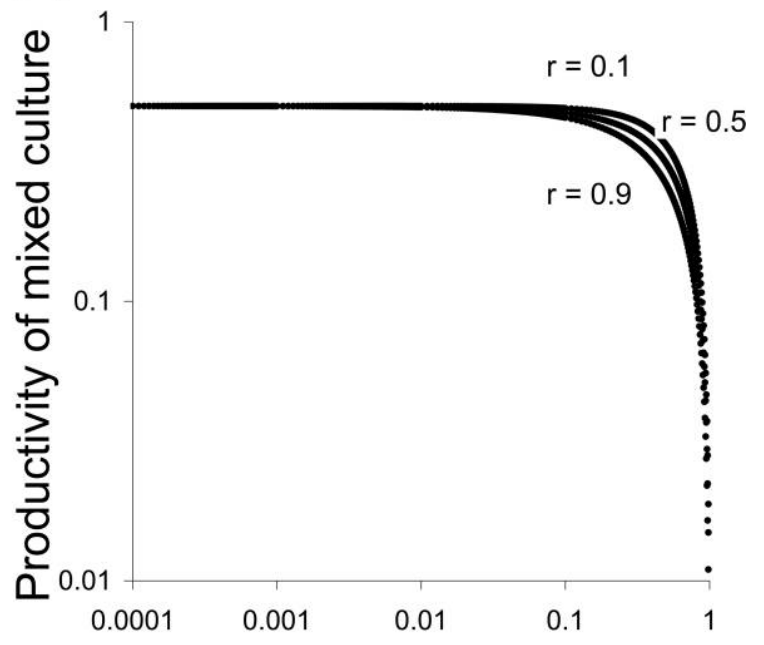

Proportion cheaters in initial population

Figure 1: Structured population model, with fitness given by the product of $g(s)=1-s / 2$ and $h(\hat{s})=\hat{s}$, where $s$ is an individual's own contribution to public goods and $\hat{s}$ is the amount of public goods in the individual's social environment. $A$, Relative fitness of cheaters $(v)$ decreases with their frequency $(p)$ and with the degree of population structure $(r) . B$, Total productivity of the population decreases with the frequency of cheaters $(p)$ and with degree of population structure $(r)$.

ative fitness is examined using the same approach. The derivative

$$
\frac{\mathrm{d} v}{\mathrm{~d} r}=-\frac{g_{\mathrm{D}}}{g_{\mathrm{C}}} \frac{p h_{\mathrm{D}} h_{\mathrm{C}}^{\prime}+(1-p) h_{\mathrm{C}} h_{\mathrm{D}}^{\prime}}{h_{\mathrm{C}}^{2}}
$$

is a negative quantity, and hence increasing population structure (higher $r$ ) reduces the relative fitness of cheaters. This is because cheaters will have a higher fitness in more mixed populations, where they are better able to exploit the cooperators. This could be tested empirically by examining the strength of frequency dependence across populations that are structured to different degrees, for example, using shaken versus unshaken liquid culture media or semisolid agar culture media of varying viscosities. $\mathrm{Nu}$ merical illustrations of the model are given in figure 1 .

\section{Population Growth as a Function of Cooperation Frequency}

Another way in which strong selection can give rise to frequency dependence is when the growth of a population depends on its genetic composition. If the growth of a bacterial population is negligible when cheating is prevalent, we expect little change in population size and genetic composition, due to cheaters having a limited opportunity to exploit cooperators. Conversely, if more cooperative bacterial colonies achieve a higher carrying capacity and hence more growth, differences in growth rates of cooperators and cheaters could lead to more pronounced changes in gene frequencies. Describing fitness in terms of absolute increase over the growth period, the relative fitness of faster growers increases with the number of rounds of division. Put simply, more growth means a greater chance for cheaters to exploit cooperators.

For simplicity, consider a well-mixed population of bacteria growing exponentially over a time period that depends on the initial proportion of cooperators. Defining a basic time unit such that the instantaneous rate of growth is 1 for cooperators, then the growth rate of cheaters can be represented as $1+b$. After $t$ time units, the numbers of cooperators and cheaters are given by

$$
\begin{aligned}
& n_{\mathrm{C}, t}=n_{\mathrm{C}, 0} e^{t}, \\
& n_{\mathrm{D}, t}=n_{\mathrm{D}, 0} e^{(1+b) t},
\end{aligned}
$$

respectively, where $n_{\mathrm{C}, 0}$ and $n_{\mathrm{D}, 0}$ are, respectively, the numbers of cooperators and cheaters at time $t=0$. If growth ceases at time $t=T\left(p_{0}\right)$, which decreases with the initial proportion of cheaters $\left(p_{0}\right.$; i.e., $\left.\mathrm{d} T / \mathrm{d} p_{0}<0\right)$, then the frequency of cheaters following growth is given by

$$
p_{T}=\frac{n_{\mathrm{D}, T}}{n_{\mathrm{D}, T}+n_{\mathrm{C}, T}}=\frac{p_{0} e^{b T}}{p_{0} e^{b T}+1-p_{0}} .
$$


(A)

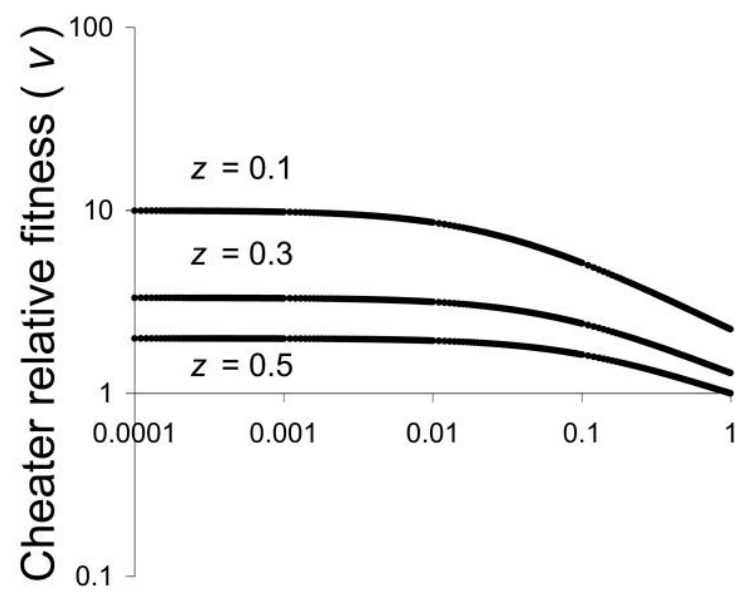

(B)

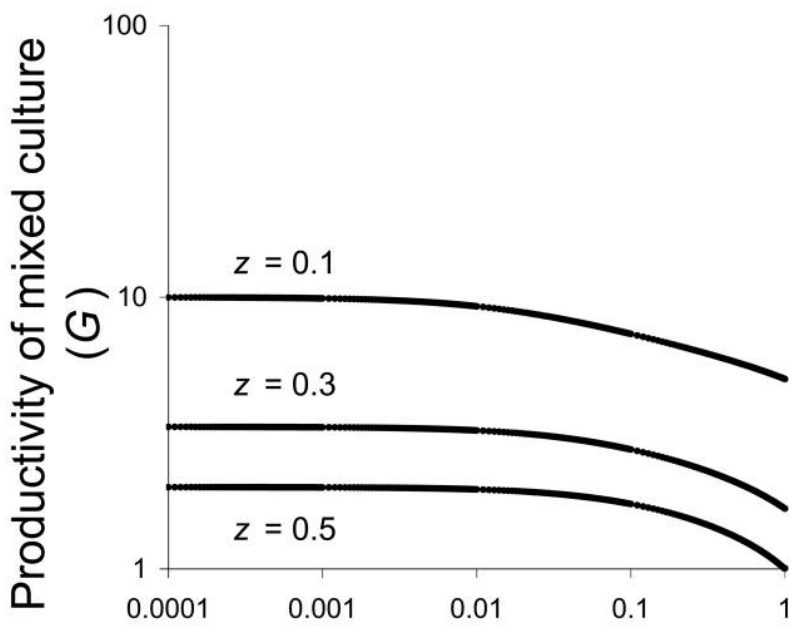

Proportion cheaters in initial population

Figure 2: Logistic growth model, with more cooperative populations experiencing higher carrying capacity, for a range of initial population sizes $\left(z_{0}=n_{\mathrm{C}, 0}+n_{\mathrm{D}, t}\right.$ as a proportion of carrying capacity for a fully cooperative population) and initial proportion of cheaters $\left(p_{0}=n_{\mathrm{D}, 0} / z_{0}\right)$. We assume that cheaters grow at twice the rate of cooperators and that they exert twice as great a competitive strain on resources $(a=b=1)$. A, Relative fitness of cheaters $(v)$ decreases as initial population size $\left(z_{0}\right)$ and initial proportion of cheaters $\left(p_{0}\right)$ increases, because both result in reduced population growth and, hence, less pronounced fitness differences. $B$, Total productivity of the population $(G)$ decreases as initial population size $\left(z_{0}\right)$ and initial proportion of cheaters $\left(p_{0}\right)$ increases, because both result in reduced population growth.

Hence, the relative fitness of cheaters versus cooperators is

$$
v=e^{b T}
$$

It is easy to show that relative fitness of cheaters is frequency dependent and, indeed, that it is a decreasing function of cheater frequency:

$$
\frac{\mathrm{d} v}{\mathrm{~d} p_{0}}=\frac{\partial v}{\partial T} \frac{\mathrm{d} T}{\mathrm{~d} p_{0}}=b e^{b T} \frac{\mathrm{d} T}{\mathrm{~d} p_{0}}
$$

is negative because $\mathrm{d} T / \mathrm{d} p_{0}<0$. Note that frequency independence is recovered in the limit of weak selection (small $b$ ). Here, we can write $T=\hat{T}+O(b)$, where $\hat{T}$ is a constant with respect to the initial frequency of cheaters, $p_{0}$. Hence, from a Taylor expansion of equation (13), relative fitness is given by $v=1+\hat{T} b+O\left(b^{2}\right)$; that is, to a first-order approximation, it is independent of the frequency of cheaters.

This model of exponential growth, with an abrupt halt at a time depending on the population level of cooperation, is unrealistic and pursued only for the sake of analytic tractability. We now consider a more realistic model describing logistic growth and competition for resources, in which a population of cooperators can maintain a higher cell density than can a population of cheaters. In particular, we continue to assume an intrinsic growth rate of 1 and $1+b$ for cooperators and cheaters, respectively, but also include a density-dependence term in the dynamical equations describing growth:

$$
\begin{aligned}
& \frac{\mathrm{d} n_{\mathrm{C}}}{\mathrm{d} t}=n_{\mathrm{C}, t}\left(1-E_{t}\right), \\
& \frac{\mathrm{d} n_{\mathrm{D}}}{\mathrm{d} t}=(1+b) n_{\mathrm{D}, t}\left(1-E_{t}\right),
\end{aligned}
$$

where the numbers of cooperators and cheaters $\left(n_{\mathrm{C}, t}\right.$ and $\left.n_{\mathrm{D}, t}\right)$ are expressed as proportions of the total population size that can be maintained at equilibrium (carrying capacity) if all cells cooperate and $E_{t}=n_{\mathrm{C}, t}+(1+a) n_{\mathrm{D}, t}$ is the "effective" population size, in terms of the strain on resources that slows population growth, where each cheating individual incurs a strain that is equivalent to that of $1+a$ cooperators. This reflects the benefit of cooperation for the population: for $a>0$, cheaters inflate the effective size of the population so that, when they are common, the actual number of cells that can be sustained at stationary phase is reduced. This model contains nonlinearities that prevent an analytical treatment, although numerical solutions are possible (fig. 2), and these recover 
the same qualitative results derived above for the simpler exponential growth model.

\section{Methods}

We experimentally tested our prediction that cheater fitness will be negatively correlated with the frequency of cheaters when population growth increases with cooperator frequency. We minimized the possible effects of structured populations leading to frequency dependence by carrying out growth in a shaken liquid media.

\section{Description of Strains}

Three Pseudomonas aeruginosa cooperator-cheater pairs (A-C) were used in this experiment. Each pair comprised a cooperator strain ("+"), a wild-type isolate that did produce pyoverdine (pvd), and a cheater strain (“-”), a pvddefective mutant derived from the cooperator strain, giving $\mathrm{A}+, \mathrm{A}-, \mathrm{B}+, \mathrm{B}-, \mathrm{C}+$, and $\mathrm{C}-$. Specifically, the strains used were as follows. A+: PA01 (strain ATCC15692), a pvd-producing wild type; A-: PAO6609 (Hohnadel et al. 1986), a pvd-negative mutant derived by ultraviolet mutagenesis from methionine auxotroph PAO6409 (Rella et al. 1985), which in turn was generated by transposon mutagenesis from PA01; B+: PA01 (strain ATCC15692), a pvd-producing wild type, as for A+ above; B-: PA2399, an unmarked deletion mutant derived from PA01, defective for the pyoverdine synthetase gene $p v d D$ (Ghysels et al. 2004); C+: a wild type, pvd-producing clone cultured from UCBPP-PA14, a clinical isolate known to also be pathogenic in plants and animals (Rahme et al. 1995); C-: a pvd-negative mutant coevolved with $\mathrm{C}+$ in the laboratory under iron-limiting conditions, which should favor the spread of spontaneously arising mutants that somehow avoid the cost of producing siderophores and instead take up those produced by neighboring bacteria. Both $\mathrm{C}+$ and $\mathrm{C}$ - were isolated from the same overnight culture, which at that point had been passaged through 19 (daily) serial transfers, where each transfer entailed the inoculation of $60 \mu \mathrm{L}$ of overnight culture (incubated at $37^{\circ} \mathrm{C}$ and shaken at $200 \mathrm{rpm}$ ) into $30-\mathrm{mL}$ glass universal vials containing 6 $\mathrm{mL}$ fresh medium (CAA; $5 \mathrm{~g}$ casamino acids, $1.18 \mathrm{~g}$ $\left.\mathrm{K}_{2} \mathrm{HPO}_{4} \cdot 3 \mathrm{H}_{2} \mathrm{O}, 0.25 \mathrm{~g} \mathrm{MgSO}_{4} .7 \mathrm{H}_{2} \mathrm{OL}^{-1}\right)$. Like $\mathrm{A}-$ and $\mathrm{B}-$, which are known to be pvd-synthesis defective, mature $\mathrm{C}$ - colonies also have a pale white appearance, distinguishing them from both $\mathrm{C}+$ and their parent strain, which appear conspicuously green owing to the presence of pyoverdine.
Experimental Design

For each strain pair, “+” and “-” cultures were initiated from freezer stock and incubated overnight in an orbital shaker $\left(37^{\circ} \mathrm{C}, 200 \mathrm{rpm}\right)$. For this first stage, strains were grown in $6 \mathrm{~mL}$ standard King's $\mathrm{B}(\mathrm{KB})$ medium in 30$\mathrm{mL}$ glass universal vials. After $24 \mathrm{~h}$, the cultures were retrieved and vortexed for $45 \mathrm{~s}$. For pair A only, pilot data predicted substantial cell density differences between the $\mathrm{A}+$ and $\mathrm{A}-$ cultures after $24 \mathrm{~h}$ growth in $\mathrm{KB}$, so to equalize cell densities in the " + " and "-" cultures, a volume of $\mathrm{A}+$ culture $(3.64 \mathrm{~mL})$ was drawn off and replaced with buffer solution (M9).

By appropriate dilution, we then prepared "treatment" cultures, mixing “-” (cheaters) with “+” (cooperators) at cell density ratios of approximately $1: 1,000,1: 100,1: 10$, $1: 1$, and $100: 1$. These cultures were supplemented with buffer (M9) such that each would contain cells at approximately the same density $\left(\sim 10^{6}\right.$ cells $\left./ 60 \mu \mathrm{L}\right)$. For each of these five treatments, we inoculated six replicate "competition" vials with $60 \mu \mathrm{L}$ of the prepared mixed cultures: 30-mL universal glass vials containing $6 \mathrm{~mL}$ CAA medium, as described previously, supplemented with $100 \mathrm{mg} \mathrm{mL}^{-1}$ human apo-transferrin (an iron chelator) and $20 \mathrm{mM}$ $\mathrm{NaHCO}_{3}$, necessary for effective chelator activity (Meyer et al. 1996). The 30 competition vials were then placed in random order in an orbital shaker for overnight incubation $\left(37^{\circ} \mathrm{C}, 200 \mathrm{rpm}\right)$.

To assess the initial ratios of cooperators to cheaters in the inocula, multiple samples were taken and grown on KB-agar plates, and colony-forming units (CFUs) were counted and categorized as either "+" or "-" on the basis of color and morphology. Similarly, each of the 30 competition cultures, after $24 \mathrm{~h}$ of incubation at $37^{\circ} \mathrm{C}$, was diluted, spread onto KB-agar plates, and incubated again overnight for counting. In this way, we obtained data on the absolute density and relative proportions of " + " and "-" CFUs both before and after the competition period. At each stage, labeling, spreading, and counting of plates were fully randomized to minimize order effects.

For pairs A and B, a single round of competition was conducted. In the case of pair $\mathrm{C}$, two rounds were conducted, in each of which a different treatment failed to yield useful data. The results of both rounds were thus pooled for combined analysis.

We also carried out a separate experiment to test whether reducing the period of population growth reduced the extent of frequency dependence. Using strain pair A, we simultaneously inoculated two parallel sets of three treatments, with cheater: cooperator ratios of approximately $1: 1,000,1: 10$, and $100: 1$. Our protocol was identical to that described above except that, whereas one set 
was cultured for a full $24 \mathrm{~h}$ before sampling, the second set was sampled after just $6 \mathrm{~h}$ of incubation.

\section{Statistical Analyses}

We performed two types of analyses on data from each of the three strain pairs. First, we calculated relative cheater fitness $(v)$, by comparing the frequency of cheaters at the beginning and end of the experiment. Specifically, $v$ is given by $v=x_{2}\left(1-x_{1}\right) / x_{1}\left(1-x_{2}\right)$, where $x_{1}$ is the initial proportion of cheaters in the population and $x_{2}$ is their final proportion. The value of $v$, therefore, signifies whether cheaters increase in frequency $(v>1)$, decrease in frequency $(v<1)$, or remain at the same frequency $(v=$ 1). We assessed the nature and strength of the relationship between $v$ and $x_{1}$, using standard general linear models (GLMs), with $x_{1}$ as the explanatory variable. In each case, $v$ and $x_{1}$ were first log transformed or, in the case of pairs 1 and 2, converted to the form $\log (v+1)$ to normalize the distribution of the residuals, in accordance with the assumptions of parametric analyses. In the case of pair C, "round" was included as a blocking factor in the maximal model but was nonsignificant and so was excluded from the final model. Second, we quantified productivity $(G)$ as the final cell density attained by the mixed culture at the end of the competition period. Again, this was analyzed with respect to initial cheater frequency, $x_{1}$, using a standard GLM. Here again, $G$ was first log transformed to normalize the distribution of the residuals. All analyses were performed with Minitab 14.1 (http://www.minitab .com).

\section{Results}

In all three cheater-cooperator pairs, the relative fitness of cheaters was significantly negatively correlated with initial cheater frequency (fig. $3 A$ ). In all cases, the relationship was best described by a power function (pair A: $v=$ 4.200 $x_{1}^{-0.442}$, GLM: $F=92.17, \mathrm{df}=1,28, P<.001$; pair B: $v=1.856 x_{1}^{-0.123}, \quad$ GLM: $\quad F=11.27, \quad \mathrm{df}=1,26, \quad P=$ .0024 ; pair C: $v=2.270 x_{1}^{-0.176}, \mathrm{GLM}: F=37.24, \mathrm{df}=$ $1,45, P<.001)$. At low frequencies, cheaters showed higher fitness than cooperators, while at high frequencies, cheater fitness was comparable to-or in the case of pair A, significantly lower than-that of cooperators.

For all three strain pairs, productivity of the mixed culture was significantly negatively correlated with initial cheater frequency (fig. $3 B$ ). In all cases, the relationship was best described by an exponential function (pair A: $G=$ $3.56 \times 10^{8} e^{-1.97 x_{1}}$, GLM: $F=74.83, \mathrm{df}=1,28, P<.001$; pair B: $G=2.82 \times 10^{8} e^{-2.38 x_{1}}$, GLM: $F=75.82, \mathrm{df}=$ 1,26, $P<.001, r^{2}=0.70$; pair C: $G=1.04 \times 10^{9} e^{-2.13 x_{1}}$, GLM: $F=139.11, \mathrm{df}=1,51, P<.001)$. In cultures initi- ated with a high frequency of cheaters (i.e., the $100: 1$ treatment), productivity was around an order of magnitude lower than in cultures where cheaters were scarce (i.e., the $1: 1,000$ treatment).

The strength of frequency dependence was reduced when populations were allowed to grow for shorter periods of time (fig. 4). In our further experiment with strain pair A, the population growth was significantly lower in the 6h-old cultures than in the 24-h-old cultures (fig. 4B; 24h cultures: $G=1.01 \times 10^{8} e^{-2.77 x_{1}}, 6$-h cultures: $G=$ $1.68 \times 10^{6} e^{3.824 x_{1}}$; GLM: final density $\times$ time $F=119.76$, $\mathrm{df}=1,29, P<.001)$. The strength of frequency dependence with respect to cheater relative fitness was also reduced over the shorter time period (fig. 4A; 24-h cultures: $v=1.303 x_{1}^{-0.241}, \quad 6$-h cultures: $v=1.117 x_{1}^{-0.036}$; GLM: fitness $\times$ time $F=13.06, \mathrm{df}=1,27, P<.001$ ).

We carried out a number of additional analyses to test the robustness of our results. First, we considered an alternative measure of productivity: the fold increase in total cell density over the competition period. These analyses produced results qualitatively concordant with those presented here. Second, we considered an alternative approach for all fitness versus proportion analyses: instead of regressing $x_{1}$ against $v$ (an expression that itself contains $\left.x_{1}\right)$, we simply regressed the initial odds ratio, $x_{1} /(1-$ $\left.x_{1}\right)$ against the final odds ratio, $x_{2} /\left(1-x_{2}\right)$. By this approach, fitted regression lines with slopes significantly less than 1 indicate negative frequency dependence. In the first set of experiments, we found this result for all three strain pairs (pair A: slope $0.73 \pm 0.02, T_{\mathrm{s}}=12.30, \mathrm{df}=28$, $P<.001$; pair B: slope $0.93 \pm 0.03, T_{\mathrm{s}}=2.32 \mathrm{df}=26$, $P=.029$; pair C: slope $0.84 \pm 0.04, T_{\mathrm{s}}=4.33, \mathrm{df}=48$, $P<.001)$. In the experiment considering different competition periods, only the 24 -h cultures showed negative frequency dependence (24-h cultures: slope $0.84 \pm 0.02$, $T_{\mathrm{s}}=7.68, \mathrm{df}=16, P<.001 ; 6$-h cultures: slope $0.97 \pm$ $\left.0.03, T_{\mathrm{s}}=1.29, \mathrm{df}=14, P=.218\right)$. Third, we repeated all analyses using the ratio of cheaters to cooperators $\left(x_{R}\right)$ as the explanatory variable, rather than the proportion of cheaters in the mixed population $\left(x_{1}\right)$, where $x_{R}=$ $x_{1} /\left(1-x_{1}\right)$. For all three strain pairs, results obtained in regressions with $x_{\mathrm{R}}$ were qualitatively analogous to those presented here.

\section{Discussion}

In this study, we have determined under what conditions the production of public goods in microbes will be subject to frequency-dependent selection. Specifically, we have shown that if there is strong selection, two sets of conditions can lead to frequency-dependent fitness: (1) when cooperators acquire more than a random benefit of public good production-for example, if cooperators tend to be 

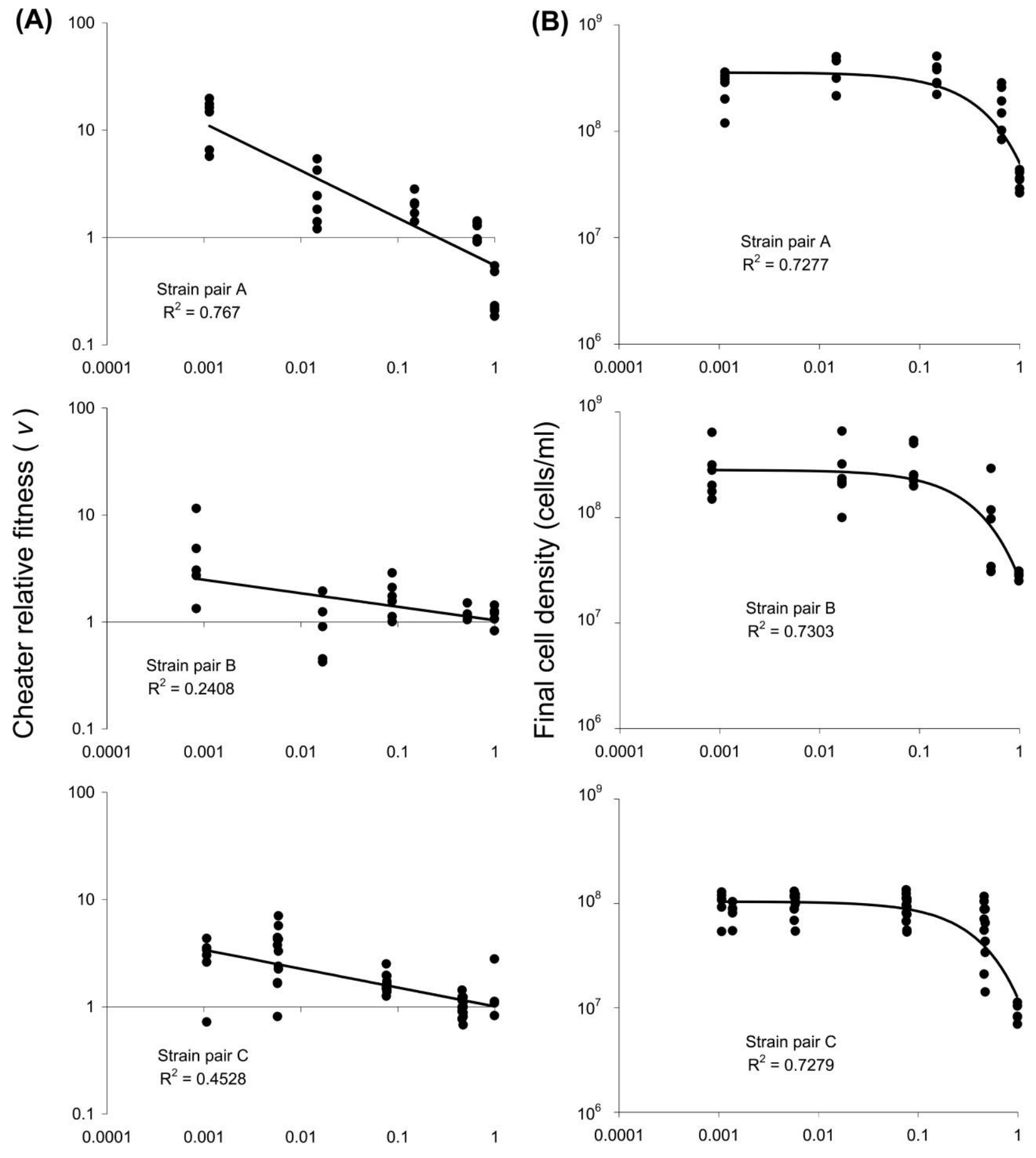

\section{Proportion cheaters in initial population}

Figure 3: Relative cheater fitness $(A)$ and mixed-culture productivity $(B)$ as functions of initial cheater frequency for three independent pairs of Pseudomonas aeruginosa strains. Here, relative fitness is the proportional increase in frequency of cheaters relative to cooperators, and productivity is the final cell density of the mixed culture after a 24-h competition period. Fitted lines reflect power $(A)$ and exponential regression $(B)$ curves estimated by least squares. 


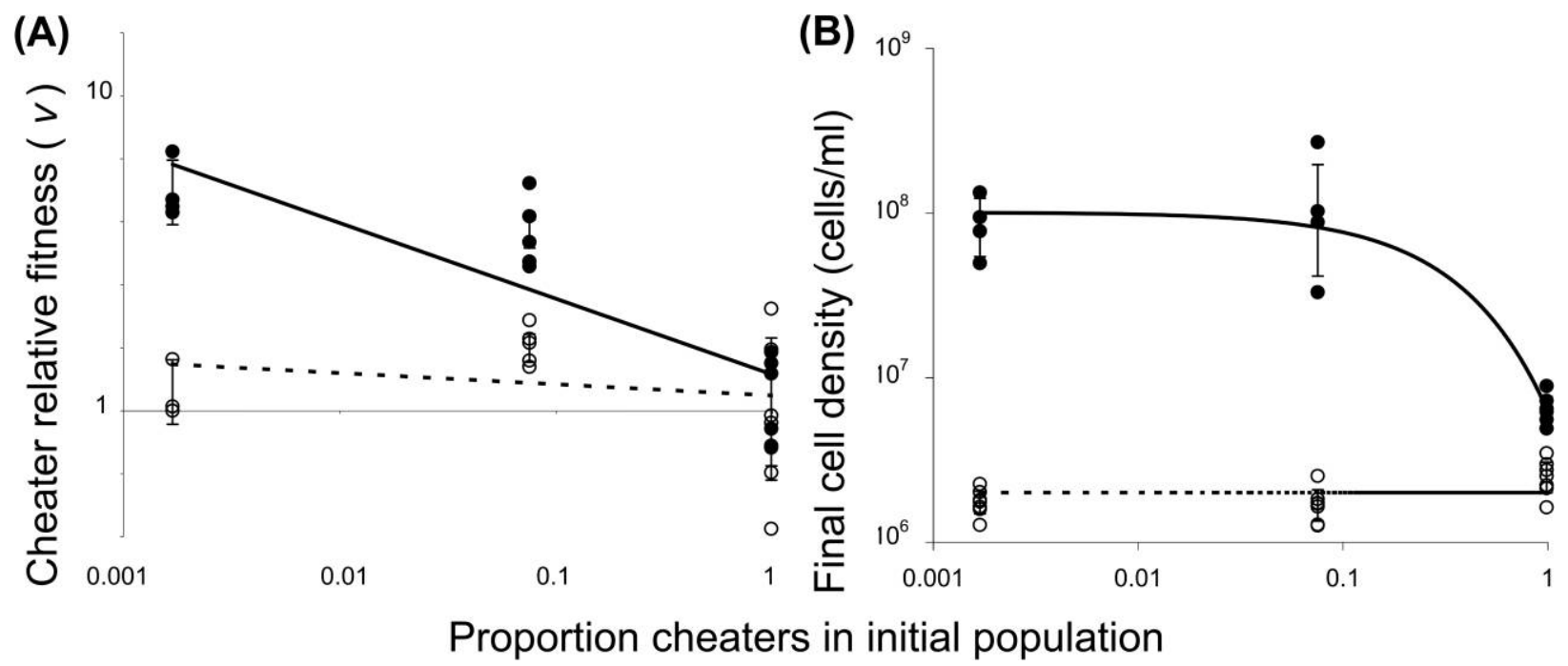

Figure 4: Relative cheater fitness $(A)$ and mixed-culture productivity $(B)$ as functions of initial cheater frequency in 6-h cultures (open circles, dotted regression line) versus 24 -h cultures (solid circles, solid regression line). Here, relative fitness is the proportional increase in frequency of cheaters relative to cooperators, and productivity is the final cell density of the mixed culture at the end of the competition period. Fitted lines reflect power $(A)$ and exponential $(B)$ regression curves estimated by least squares. Error bars reflect $95 \%$ confidence intervals around the means.

clustered together (population structuring) or if individual cells have preferential access to the public goods that they produce (fig. 1); or (2) when increased levels of cooperation lead to greater growth, a higher carrying capacity, and hence more generations over which cheaters can exploit cooperators (fig. 2). In both cases, the relative fitness of cooperators or cheaters is greater when they are less common. We then explicitly tested the second of these possibilities (by controlling for the first, population structuring), using the production of iron-scavenging siderophore molecules in Pseudomonas aeruginosa as a model trait. As predicted, we found a negative relationship between the relative fitness of cheaters and the proportion of cheaters in the population (fig. $3 A$ ). Furthermore, we found support for the underlying assumption leading to this prediction-a higher proportion of cooperation led to greater growth (fig. $3 B$ ), and a reduction in the period of growth led to a decrease in the extent of frequency dependence (fig. 4).

\section{Situations Favoring Frequency-Dependent Selection}

We have identified two ways in which kin selection models of public goods dilemmas can generate frequency-dependent selection, given strong selection. First, population structure, incorporating both limited mixing of cells and limited diffusion of public goods molecules (local social interaction) can result in the selective advantage of cheating decreasing as cheaters become more common. When populations are structured, the level of public goods available to an individual will depend on (1) the production of public goods by that individual and its clonal relatives and (2) the average level of public goods production in the population. Increasing population structure increases the importance of the former and decreases the importance of the latter. A higher frequency of cheaters will lead to a lower average level of public goods production (2). The consequences of this will be greater for cheaters, because they receive fewer public goods through their own production and that of their relatives (a). This leads to the relative fitness of cheaters decreasing with cheater frequency. Second, frequency dependence may also arise when total population growth increases with the level of cooperation in the population. If more cooperative populations achieve a higher carrying capacity, then more rounds of growth and division are possible before stationary phase is reached. The selective benefit of cheating multiplies with each round of division, so that the relative fitness of a cheater measured over the period of population expansion increases with the (initial) frequency of cooperators in the population (fig. $2 A$ ).

How does our work relate to previous theory? We have provided some specific cases for the general conclusion that the generation of frequency-dependent selection relies on strong selection (Charlesworth 1978; Michod 1982; Toro et al. 1982; Queller 1984; Nowak and May 1992; Day and Taylor 1998; Rousset 2004; Jansen and Van Baalen 2006). The aim of this previous work was to elucidate general 
points, and so scenarios were modeled that do not facilitate simple application to specific systems such as public goods production (e.g., two-player games; Queller 1984). An important exception to this was provided by Dugatkin et al. (2003), who explicitly considered frequency-dependent selection in microbes. However, Dugatkin et al. (2003) did not explain how frequency dependence should occur in the first place-their aim was to address the consequences of frequency-dependent selection, and so they simply assumed that it occurred. This is analogous to taking equation (7) or (13) as a starting point rather than determining how they should arise. More generally, frequency dependence does not usually arise from most of social evolution theory because selection is usually assumed to be weak, to make analyses more tractable (Hamilton 1964; Taylor and Frank 1996; Frank 1998; Rousset 2004). Frequency dependence can also arise in models for the evolution of cooperation that do not rely on kin selection (e.g., reciprocity; Axelrod and Hamilton 1981).

\section{Frequency-Dependent Selection in Microbes}

Frequency-dependent selection can, under certain conditions, prevent cheater takeover and facilitate the coexistence of multiple phenotypes (Aviles 2002). However, in two of the three strain pairs examined in this study, cheaters were at least as fit as cooperators, even when common (fig. 3A). With such a payoff structure, we may anticipate that, in time, cheaters would go to fixation in populations where there is little competition between groups. Consistent with this idea, siderophore-defective strains are routinely isolated from the lungs of late-stage cystic fibrosis patients, where they typically grow as monoclonal populations (Lee et al. 2005; Salunkhe et al. 2005; Smith et al. 2006). On the other hand, in an environment where competition occurs between subpopulations and these subpopulations contain different proportions of cheaters, those groups with more cooperators may outcompete cheater-dominated groups (Griffin et al. 2004). Thus, cooperation may also be maintained by frequency-dependent selection at the level of the group.

We suggest that negative frequency dependence should be common in microbes, because (1) both population structuring and cooperation-dependent growth rates are likely to be important in many situations, and (2) mutations can occur that lead to large differences in the level of cooperation (strong selection; see below). Accordingly, negative frequency-dependent selection has now been shown for social cheaters in at least 12 studies on seven microbial species (Velicer et al. 2000; Vulic and Kolter 2001; Fiegna and Velicer 2003; Rainey and Rainey 2003; Turner and Chao 2003; Dugatkin et al. 2005; Brockhurst et al. 2006; Harrison et al. 2006; MacLean and Gudelj 2006;
Ellis et al. 2007; Gilbert et al. 2007; this study). However, while we highlight these studies as support for our prediction, we also point out that most did not explicitly test the underlying assumptions of why frequency dependence occurs. Ultimately, a range of complementary approaches is most useful. At one extreme will be studies designed to reveal those specific mechanisms responsible for frequency dependence. For example, our aim in this study was to test one particular mechanism that can lead to frequency dependence-cooperation-dependent population growth. In order to do this, we (1) carried out our experiment in shaken vials, in order to remove the other possible mechanism that can lead to frequency-dependent selectionpopulation structure - and (2) tested the underlying assumption that a higher proportion of cooperators allows the population to grow to a larger size (fig. $3 B$ ). At the other extreme are studies in more natural conditions that examine the net consequences of both mechanisms for generating frequency dependence (e.g., Harrison et al. 2006). An important task for the future is to determine the relative importance in nature of the two different mechanisms that we have shown can lead to frequency dependence.

We observed a decrease in the extent of frequency dependence when the period of time available for growth was shortened (fig. 4). This supported the assumption of our model that the relative fitness of cheats increases over time as the population goes through more rounds of growth and division. It is possible that other factors could also cause the relative fitness of cheaters to vary over time. For example, as the population grows, this could lead to a decrease in iron availability that reduces the relative fitness of cheaters (Griffin et al. 2004) or a higher population density that allows cheaters to better exploit cooperators and hence increases their own relative fitness (Greig and Travisano 2004; MacLean and Gudelj 2006). However, effects such as these cannot explain the frequency dependence evident in our data; in order for frequency dependence to be observed, one of the additional factors that we have suggested would still be required, such as populations with greater frequencies of cooperators growing to higher final densities.

More generally, our study illustrates that, although the same social evolution theory originally developed for metazoans can be applied to microbes, certain aspects of microbial biology mean that some differences may occur in the evolution of social traits. In particular, with typical social evolution study organisms, such as insects, birds, and mammals, it is usually assumed that mutation will lead to minor variations in behavior (weak selection), and so frequency dependence will be relatively unimportant (Sinervo and Lively 1996; Sinervo et al. 2006; Sinervo and Calsbeek 2006). In contrast, in the case of microbes, single 
or small numbers of mutations can have extremely large effects on social behaviors (strong selection; Velicer et al. 1998, 2000; Rainey and Rainey 2003; Velicer and Yu 2003; Foster et al. 2004; Griffin et al. 2004; Fiegna et al. 2006). For example, in $P$. aeruginosa, social behaviors such as siderophore production are routinely and completely lost from isolates obtained from lung infections (Lee et al. 2005; Salunkhe et al. 2005; Smith et al. 2006). Furthermore, the majority of microbial cooperative behaviors are controlled by quorum sensing regulatory networks (Keller and Surette 2006; Venturi 2006; Diggle et al. 2007). Consequently, the disruption of these networks, even through the loss of a single gene, can have huge effects on the level of cooperation. A consequence of the potential for mutations of large effects (strong selection) in microbes, is that this allows frequency-dependent selection, through the mechanisms we have described in this study.

\section{Acknowledgments}

We thank A. Buckling and P. Cornelis for providing bacterial strains, B. Evans for laboratory assistance, and N. Colegrave, R. Kümmerli, G. Wild, and an anonymous reviewer for discussion and comments on the manuscript.

\section{Literature Cited}

Aviles, L. 2002. Solving the freeloaders paradox: genetic associations and frequency-dependent selection in the evolution of cooperation among nonrelatives. Proceedings of the National Academy of Sciences of the USA 99:14268-14273.

Axelrod, R., and W. D. Hamilton. 1981. The evolution of cooperation. Science 211:1390-1396.

Brockhurst, M. A., M. E. Hochberg, T. Bell, and A. Buckling. 2006. Character displacement promotes cooperation in bacterial biofilms. Current Biology 16:2030-2034.

Brown, S. P. 1999. Cooperation and conflict in host-manipulating parasites. Proceedings of the Royal Society B: Biological Sciences 266:1899-1904.

Charlesworth, B. 1978. Some models of the evolution of altruistic behaviour between siblings. Journal of Theoretical Biology 72:297319.

Crespi, B. J. 2001. The evolution of social behaviour in microorganisms. Trends in Ecology \& Evolution 16:178-183.

Day, T., and P. D. Taylor. 1998. Unifying genetic and game theoretic models of kin selection for continuous traits. Journal of Theoretical Biology 194:391-407.

Diggle, S. P., A. Gardner, S. A. West, and A. S. Griffin. 2007. Evolutionary theory of bacterial quorum sensing: when is a signal not a signal? Philosophical Transactions of the Royal Society B: Biological Sciences 362:1241-1249.

Dugatkin, L. A., M. Perlin, and R. Atlas. 2003. The evolution of group-beneficial traits in the absence of between-group selection. Journal of Theoretical Biology 220:67-74.

Dugatkin, L. A., M. Perlin, J. S. Lucas, and R. Atlas. 2005. Groupbeneficial traits, frequency-dependent selection and genotypic di- versity: an antibiotic resistance paradigm. Proceedings of the Royal Society B: Biological Sciences 272:79-83.

Ellis, R. J., A. K. Lilley, S. J. Lacey, D. Murrell, and H. C. J. Godfray. 2007. Frequency-dependent advantages of plasmid carriage by Pseudomonas in homogeneous and spatially structured environments. ISME Journal 1:92-95.

Fiegna, F., and G. J. Velicer. 2003. Competitive fates of bacterial social parasites: persistence and self-induced extinction of Myxococcus xanthus cheaters. Proceedings of the Royal Society B: Biological Sciences 270:1527-1534.

Fiegna, F., Y. T. Yu, S. V. Kadam, and G. J. Velicer. 2006. Evolution of an obligate social cheater to a superior cooperator. Nature 441: 310-314.

Foster, K. R., G. Shaulsky, J. E. Strassmann, D. C. Queller, and C. R. Thompson. 2004. Pleiotropy as a mechanism to stabilize cooperation. Nature 431:693-696.

Frank, S. A. 1998. Foundations of social evolution. Princeton University Press, Princeton, NJ.

- 2003. Repression of competition and the evolution of cooperation. Evolution 57:693-705.

Ghysels, B., B. T. Dieu, S. A. Beatson, J. P. Pirnay, U. A. Ochsner, M. L. Vasil, and P. Cornelis. 2004. Fpvb, an alternative type I ferripyoverdine receptor of Pseudomonas aeruginosa. Microbiology 150:1671-1680.

Gilbert, O. M., K. R. Foster, N. J. Mehdiabadi, J. E. Strassman, and D. C. Queller. 2007. High relatedness maintains multicellular cooperation in a social amoeba by controlling cheater mutants. Proceedings of the National Academy of Sciences of the USA 104: 8913-8917.

Greig, D., and M. Travisano. 2004. The Prisoner's Dilemma and polymorphism in yeast SUC genes. Proceedings of the Royal Society B: Biological Sciences 271(suppl.):25-26.

Griffin, A. S., S. A. West, and A. Buckling. 2004. Cooperation and competition in pathogenic bacteria. Nature 430:1024-1027.

Guerinot, M. L. 1994. Microbial iron transport. Annual Review of Microbiology 48:743-772.

Hamilton, W. D. 1964. Genetical evolution of social behaviour. Journal of Theoretical Biology 7:1-52.

1988. This week's citation classic. Current Contents 40:16.

- 1996. Narrow roads of gene land. Vol. 1. Evolution of social behaviour. W. H. Freeman, Oxford.

Harrison, F., L. E. Browning, M. Vos, and A. Buckling. 2006. Cooperation and virulence in acute Pseudomonas aeruginosa infections. BMC Biology 4:21.

Hohnadel, D., D. Haas, and J. M. Meyer. 1986. Mapping of mutations affecting pyoverdine production in Pseudomonas aeruginosa. FEMS Microbiology Letters 36:195-199.

Jansen, V. A. A., and M. Van Baalen. 2006. Altruism through beard chromodynamics. Nature 440:663-666.

Keller, L., and M. G. Surette. 2006. Communication in bacteria: an ecological and evolutionary perpsective. Nature Reviews Microbiology 4:249-258.

Lee, B., J. A. J. Haagensen, O. Ciofu, J. B. Andersen, N. Hoiby, and S. Molin. 2005. Heterogeneity of biofilms formed by nonmucoid Pseudomonas aeruginosa isolates from patients with cystic fibrosis. Journal of Clinical Microbiology 43:5247-5255.

Lehmann, L., and L. Keller. 2006. The evolution of cooperation and altruism: a general framework and a classification of models. Journal of Evolutionary Biology 19:1365-1376. 
Leimar, O., and P. Hammerstein. 2006. Facing the facts. Journal of Evolutionary Biology 19:1403-1405.

Levin, B. R. 1988. Frequency-dependent selection in bacterial populations. Philosophical Transactions of the Royal Society B: Biological Sciences 319:459-472.

MacLean, R. C., and I. Gudelj. 2006. Resource competition and social conflict in experimental populations of yeast. Nature 441:498-501.

Maynard Smith, J., and E. Szathmàry. 1995. The major transitions in evolution. W. H. Freeman, Oxford.

Meyer, J. M., A. Neely, A. Stintzi, C. Georges, and I. A. Holder. 1996. Pyoverdin is essential for virulence of Pseudomonas aeruginosa. Infection and Immunology 64:518-523.

Michod, R. E. 1982. The theory of kin selection. Annual Review of Ecology and Systematics 13:23-55.

Nowak, M. A., and R. M. May. 1992. Evolutionary games and spatial chaos. Nature 359:826-829.

Queller, D. C. 1984. Kin selection and frequency dependence: a game theoretic approach. Biological Journal of the Linnean Society 23: 133-143.

Rahme, L. G., E. J. Stevens, S. F. Wolfort, J. Shao, R. G. Tompkins, and F. M. Ausubel. 1995. Common virulence factors for bacterial pathogenicity in plants and animals. Science 268:1899-1902.

Rainey, P. B., and K. Rainey. 2003. Evolution of cooperation and conflict in experimental bacterial populations. Nature 425:72-74.

Ratledge, C., and L. G. Dover. 2000. Iron metabolism in pathogenic bacteria. Annual Review of Microbiology 54:881-941.

Rella, M., A. Mercenier, and D. Haas. 1985. Transposon insertion mutagenesis of Pseudomonas aeruginosa with a tn5 derivative: application to physical mapping of the arc gene cluster. Gene 33: 293-303.

Rousset, F. 2004. Genetic structure and selection in subdivided populations. Monographs in population biology. Princeton University Press, Princeton, NJ.

- 2006. Separation of time scales, fixation probabilities and convergence to evolutionarily stable states under isolation by distance. Theoretical Population Biology 69:165-179.

Sachs, J. L., U. G. Mueller, T. P. Wilcox, and J. J. Bull. 2004. The evolution of cooperation. Quarterly Review of Biology 79:135160.

Salunkhe, P., C. H. Smart, J. A. Morgan, S. Panagea, M. J. Walshaw, C. A. Hart, R. Geffers, B. Tummler, and C. Winstanley. 2005. A cystic fibrosis epidemic strain of Pseudomonas aeruginosa displays enhanced virulence and antimicrobial resistance. Journal of Bacteriology 187:4908-4920.

Sinervo, B., and R. Calsbeek. 2006. The developmental, physiological, neural and genetic causes and consequences of frequency-dependent selection in the wild. Annual Review of Ecology, Evolution, and Systematics 37:581-610.

Sinervo, B., and C. M. Lively. 1996. The rock-paper-scissors game and the evolution of alternative male strategies. Nature 380:240243.
Sinervo, B., A. Chaine, J. Clobert, R. Calsbeek, L. Hazard, L. Lancaster, A. G. McAdam, S. Alonzo, G. Corrigan, and M. E. Hochberg. 2006. Self-recognition, color signals and cycles of greenbeard mutualism and altruism. Proceedings of the National Academy of Sciences of the USA 103:7372-7377.

Smith, E. E., D. G. Buckley, Z. Wu, C. Saenphimmachak, L. R. Hoffman, D. A. D’Argenio, S. I. Miller, et al. 2006. Genetic adaptation by Pseudomonas aeruginosa to the airways of cystic fibrosis patients. Proceedings of the National Academy of Sciences of the USA 103: 8487-8492.

Smith, J. 2001. The social evolution of bacterial pathogenesis. Proceedings of the Royal Society B: Biological Sciences 268:61-69.

Taylor, P. D., and S. A. Frank. 1996. How to make a kin selection model. Journal of Theoretical Biology 180:27-37.

Toro, M., R. Abugov, B. Charlesworth, and R. E. Michod. 1982. Exact versus heuristic models of kin selection. Journal of Theoretical Biology 97:699-713.

Turner, P. E., and L. Chao. 2003. Escape from Prisoner's Dilemma in RNA phage $\Phi 6$. American Naturalist 161:497-505.

Velicer, G. J., and Y. T. Yu. 2003. Evolution of novel cooperative swarming in the bacterium Myxococcus xanthus. Nature 425:7578.

Velicer, G. J., L. Kroos, and R. E. Lenski. 1998. Loss of social behaviors by Myxococcus xanthus during evolution in an unstructured habitat. Proceedings of the National Academy of Sciences USA 95: 12376-12380.

2000. Developmental cheating in the social bacterium Myxococcus xanthus. Nature 404:598-601.

Venturi, V. 2006. Regulation of quorum sensing in Pseudomonas. FEMS Microbiology Reviews 30:274-291.

Vulic, M., and R. Kolter. 2001. Evolutionary cheating in Escherichia coli stationary phase cultures. Genetics 158:519-526.

West, S. A., and A. Buckling. 2003. Cooperation, virulence and siderophore production in bacterial parasites. Proceedings of the Royal Society B: Biological Sciences 270:37-44.

West, S. A., E. T. Kiers, E. L. Simms, and R. F. Denison. 2002. Sanctions and mutualism stability: why do rhizobia fix nitrogen? Proceedings of the Royal Society B: Biological Sciences 269:685694.

West, S. A., A. S. Griffin, A. Gardner, and S. P. Diggle. 2006. Social evolution theory for microorganisms. Nature Reviews Microbiology 4:597-607.

West, S. A., A. S. Griffin, and A. Gardner. 2007. Social semantics: altruism, cooperation, mutualism, strong reciprocity and group selection. Journal of Evolutionary Biology 20:415-432.

Wild, G., and A. Traulsen. 2007. The different limits of weak selection and the evolutionary dynmics of finite populations. Journal of Theoretical Biology 247:382-390.

Associate Editor: Thomas N. Sherratt Editor: Michael C. Whitlock 